\title{
Satisfacción del servicio de transporte público en los estudiantes universitarios
}

\section{Satisfaction with public transportation service among university students}

\section{Satisfação do serviço de transporte público em estudantes universitários}

\author{
Omar Alejandro Pérez Cruz \\ Universidad de Colima, México \\ omar_perez@ucol.mx \\ https://orcid.org/0000-0003-3367-8259 \\ Rogelio Pinto Pérez \\ Universidad de Colima, México \\ rogepipe@ucol.mx \\ https://orcid.org/0000-0003-30579-6602
}

\section{Resumen}

El objetivo de esta investigación fue estimar la satisfacción de estudiantes universitarios con respecto a los elementos de calidad de servicio del transporte público urbano del estado de Colima. El indicador de calidad del servicio se diseñó en una encuesta de percepción de 13 ítems que incorporó una serie de aspectos del servicio. El análisis factorial se aplicó a través de una regresión logística tipo logit que permitió identificar los constructos latentes en esta escala. Se identificaron ocho constructos asociados a la calidad percibida del servicio de autobús: horario congruente, precio accesible, personal inspira confianza, servicio cubre las necesidades, tiempo de espera, distancia del recorrido, infraestructura carretera y planeación del recorrido. Estos constructos latentes se exploraron mediante una apreciación de cómo las percepciones hacia la calidad del servicio de autobús varían a través de las cohortes de sexo y ciudad para explicar el grado en que estas percepciones son de utilidad para explicar la variación en la satisfacción percibida con dicho servicio. Los resultados del análisis sugieren que las percepciones asociadas a la calidad del servicio de autobús varían significativamente entre los distintos grupos de 


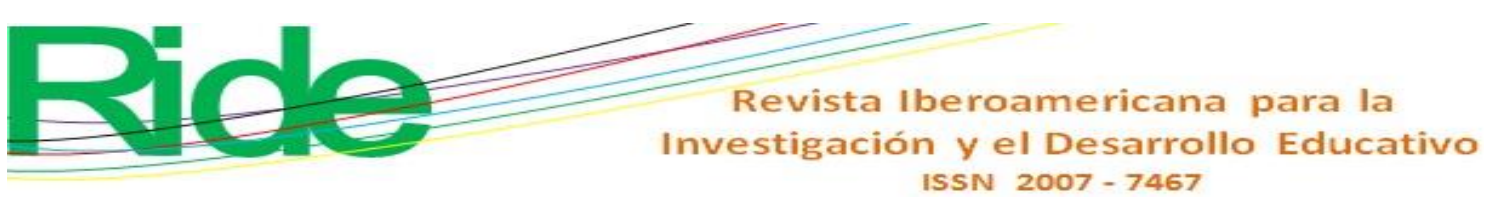

pasajeros, siendo las mujeres las que tienden a mostrar un aumento de la percepción de la calidad del servicio que pagan por el TP (1.12 más veces que los hombres). Esto implica que los hombres son más exigentes que las mujeres con respecto al servicio de TP de pagan. La satisfacción en relación con el precio pagado y el servicio recibido es menor en los hombres que en las mujeres. Además, la conveniencia percibida del servicio de autobús parece tener un poder explicativo positivo significativo sobre la satisfacción percibida con el servicio de autobús, lo que sugiere que las mejoras en el horario, el precio, el personal, cubrir las necesidades, el tiempo, la distancia, la infraestructura y la planeación del recorrido probablemente aumentarán la satisfacción percibida entre los pasajeros. Como limitantes se puede señalar que los participantes de los cuales se obtuvo la percepción del servicio de transporte fueron exclusivamente estudiantes de educación media y superior. Aun así, esta investigación demostró el nivel de conocimiento adicional que puede obtenerse a través de un análisis más detallado de los datos generados sobre la política de transporte público y de los servicios ofertados a la comunidad estudiantil.

Palabras clave: análisis multivariado, estudiante universitario, servicio, transporte público.

\section{Abstract}

The objective of this research was to estimate the satisfaction of university students with respect to the service quality elements of urban public transportation in the State of Colima. The service quality indicator was designed in a 13-item perception survey that incorporated a series of service aspects. The factor analysis was applied through a logistic regression type logit allowed identifying the latent constructs that are present in this scale. Eight latent constructs associated with the perceived quality of the bus service were identified: congruent schedule, accessible price, staff inspires confidence, service meets needs, waiting time, travel distance, road infrastructure and route planning. These latent constructs were explored through an appreciation of how perceptions of bus service quality vary across sex and city cohorts to explain the degree to which these perceptions are useful in explaining variation in perceived satisfaction with bus service. The results of the analysis suggested that perceptions regarding bus service quality vary significantly across different groups of riders, with women tending to show increased perceptions of service quality paying for PT 1.12 more times than a man. This implies that men are more demanding than women with respect to the PT service they pay for. Satisfaction in relation to the price paid and the service received is lower in men than in women. In addition, 


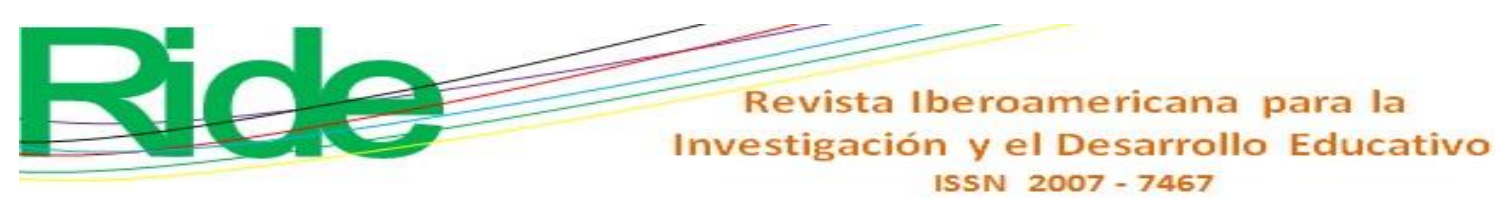

perceived convenience of bus service appears to have significant positive explanatory power on perceived satisfaction with bus service, suggesting that improvements in schedule, price, staffing, meeting needs, time, distance, infrastructure, and route planning are likely to increase perceived satisfaction among passengers.

As limitations, it was observed that the participants from whom the perception of the transportation service was obtained were exclusively middle and high school students. These results allowed us to contribute to the feedback and decision making process to improve the services offered to the student community. On a more general level, this research demonstrated the level of additional knowledge that can be gained through a more detailed analysis of the data generated on public transportation policy and services offered to the student community.

Keywords: multivariate analysis, university students, services, public transport.

\section{Resumo}

O objetivo desta pesquisa foi estimar a satisfação de estudantes universitários com relação à qualidade dos elementos de serviço do transporte público urbano no estado de Colima. O indicador de qualidade do serviço foi desenhado em uma pesquisa de percepção de 13 itens que incorporou uma série de aspectos do serviço. A análise fatorial foi aplicada por meio de uma regressão logística do tipo logit que permitiu a identificação dos construtos latentes nessa escala. Oito construtos associados à qualidade percebida do serviço de ônibus foram identificados: cronograma consistente, preço acessível, equipe que inspira confiança, serviço atende às necessidades, tempo de espera, distância da viagem, infraestrutura rodoviária e planejamento de rotas. Esses construtos latentes foram explorados por meio de uma apreciação de como as percepções em relação à qualidade do serviço de ônibus variam entre os grupos de sexo e cidade para explicar o grau em que essas percepções são úteis para explicar a variação na satisfação percebida com o referido serviço de ônibus. Os resultados da análise sugerem que as percepções associadas à qualidade do serviço de autocarro variam significativamente entre os diferentes grupos de passageiros, sendo as mulheres que tendem a apresentar um aumento na percepção da qualidade do serviço que pagam pelo PT (1,12 mais vezes do que os homens). Isto implica que os homens são mais exigentes do que as mulheres no que diz respeito ao serviço de PT pago. A satisfação em relação ao preço pago e ao serviço recebido é menor nos homens do que nas mulheres. Além disso, a conveniência percebida do serviço de ônibus parece ter um poder explicativo positivo significativo sobre a satisfação percebida com o serviço de ônibus, 


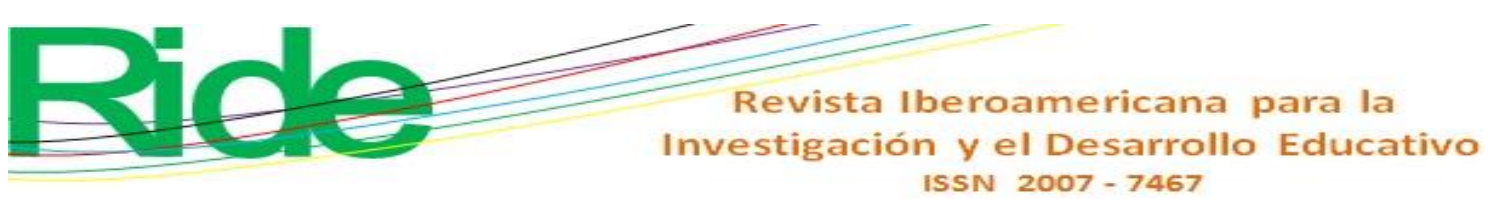

sugerindo que as melhorias no horário, preço, pessoal, necessidades de atendimento, tempo, distância, infraestrutura e planejamento de rotas provavelmente aumentarão satisfação percebida entre os passageiros. Como limitações, pode-se notar que os participantes dos quais se obteve a percepção do serviço de transporte eram exclusivamente estudantes do ensino médio e superior. Mesmo assim, esta pesquisa demonstrou o nível de conhecimento adicional que pode ser obtido por meio de uma análise mais detalhada dos dados gerados sobre a política de transporte público e os serviços oferecidos à comunidade estudantil.

Palavras-chave: análise multivariada, estudante universitário, serviço, transporte público.

Fecha Recepción: Febrero 2021

Fecha Aceptación: Agosto 2021

\section{Introducción}

En el contexto de las empresas de servicios, la satisfacción puede ser cuantificada por la percepción que el usuario tiene del propio servicio. No obstante, esta calificación solo es un elemento tangible de un cúmulo de factores ligados principalmente a la estructura del sistema en sus niveles técnico, funcional y de percepción (Duque y Gómez, 2015).

Por lo anterior, el establecimiento de los objetivos del transporte colectivo (nivel técnico) y la forma de operar, regular y concesionar el servicio (nivel funcional) son determinantes en la percepción del desempeño del sistema. De este modo, para evaluar la satisfacción del servicio es preciso medirlo de manera integral, ya que incide en al menos dos aspectos: la elección y la competencia del servicio colectivo de transporte.

El primer aspecto se refiere a la elección de los usuarios para el uso del medio de transporte público disponible. Ello - en un contexto donde el precio, los tiempos y las distancias son factores de decisión - es más probable que la satisfacción de servicio sea el factor decisivo para el modo de transporte (Subramaniana, Gunasekaran, Yu, Cheng y Ning, 2014).

En el caso de México — como en el resto de Latinoamérica - la prestación de los servicios de transporte público se concesiona a la iniciativa privada, por lo cual varios tipos de servicios de transporte colectivo o individual ofrecen la misma ruta. Esto genera la competencia entre los concesionarios del transporte público, pues la oferta se divide en varias opciones, es decir, taxis pertenecientes a un propietario, autobuses de otra empresa, etc., lo que obliga a desarrollar estrategias para atraer a la mayor cantidad de usuarios (Sánchez-Flores y RomeroTorres, 2010). 


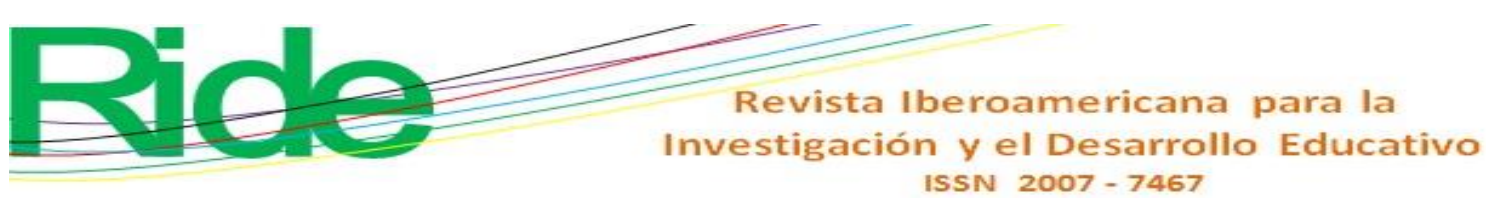

consistente con la cobertura del servicio, lo agradable de la estancia, el trato y atención del personal y el espacio suficiente y adecuado.

\section{Desarrollo}

En la literatura académica se pueden identificar tres tipos de perspectivas teóricas que distinguen los factores relacionados a la satisfacción del servicio en el transporte colectivo: la medición del desempeño del servicio, la valoración del usuario por medio de encuestas y la econometría de elección discreta con una función de utilidad.

La primera perspectiva se basa en la evaluación del desempeño del nivel de servicio (Rodríguez y Díaz, 2014) que ofrece un determinado factor. Tal desempeño se determina desde la perspectiva de algunos actores, como son los operadores y los usuarios, de forma tal que cada actor define y pondera los factores percibidos como importantes. En el caso de Latinoamérica (Aparicio, Ramírez y Gómez, 2013; Rodríguez y Díaz, 2014), algunos estudios han definido diversos factores integrados a su vez de múltiples criterios, como son tiempo de espera, tiempo de recorrido, distancia de recorrido, espacio de las unidades, seguridad de la conducción, impacto ambiental, planeación y cobertura de las rutas, entre otros.

La segunda perspectiva se basa en la valoración por medio de encuestas de la satisfacción del usuario y la identificación de brechas de calidad entre las expectativas y los servicios ofertados. En esta perspectiva el indicador de satisfacción de servicio se basó en el empleado por el gobierno de Escocia para el sector de autotransportes, el cual utiliza 11 factores para medir la calidad del servicio: tiempo de traslado; frecuencia; ajuste a las necesidades; servicio estable y sin cambios regulares; limpieza; confort; seguridad; facilidad de elegir el tipo de boleto; conocimiento de rutas y horarios; facilidad de cambiar de ruta y otros transportes, y valor de las tarifas (Morton, Caulfield y Anable, 2016).

La última perspectiva utiliza la teoría microeconómica del consumidor para crear, a partir de la especificación de un modelo de elección discreta, donde se experimentó con una función de utilidad integrada para estimar las preferencias de los viajeros y obtener una medida general de la satisfacción del servicio (Román et al., 2014). Las estimaciones obtenidas evidencian la ponderación de cada uno de los factores definidos, lo que posibilita la valoración económica (Stathopoulos y Marcucci, 2014). El análisis de los factores de satisfacción del servicio realizado a lo largo del presente artículo se circunscribe en esta última perspectiva.

La problemática del transporte público de tipo colectivo urbano en los cuatro municipios con mayor población en el estado de Colima (Tecomán, Manzanillo y la zona conurbada 


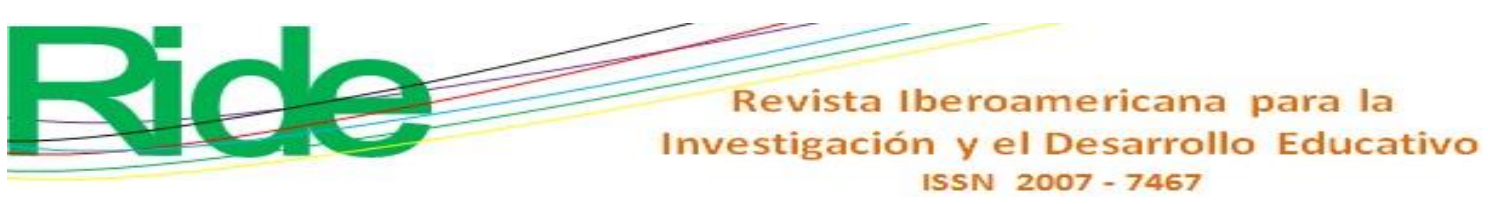

Colima-Villa de Álvarez) se puede dimensionar en una serie de factores con características casi idénticas entre ellos. La problemática económico-social del transporte urbano en esta entidad no radica en la tarifa, sino en diversos factores como el tiempo, la distancia, la cobertura, la calidad y la operatividad.

En lo referente al factor tiempo-distancia, recorrer una distancia promedio de 6 kilómetros lleva un aproximado de 55 minutos, sin considerar el tiempo de espera por no cumplir con la demanda del servicio en horarios pico. En cuanto a la cobertura, cuando más es requerido el servicio por el estudiantado menos disponibilidad existe para llegar a tiempo a los planteles de estudio.

En relación con el factor de la calidad, existe un desgaste físico exageradamente notorio en el exterior e interior de los camiones que se hallan actualmente en operación, sin tomar en cuenta que en la Ley de Movilidad Sustentable del estado de Colima (LMSEC), en su artículo 201, se establece que los autobuses del servicio de corredor urbano no deben superar los 10 años de antigüedad, prorrogables hasta 5 años (la mayoría supera los 12 años) (Periódico Oficial del Estado de Colima [POEC], 2017).

La operatividad, relacionada con el personal encargado del manejo de los camiones, se atribuye el poder de decisión de otorgar el descuento y —en un gran número de casos- el servicio a los estudiantes sin razón fundamentada alguna.

Por otra parte, los problemas de fondo que paralizan el correcto funcionamiento de este servicio público son la deficiencia administrativa, pues no existe un control estricto sobre los requerimientos para otorgar una concesión a quien en realidad la necesita; esto significa que los criterios seleccionados son decididos regularmente de manera discrecional, unipersonal y unilateral por parte de las autoridades con poder de decisión.

En segundo lugar, al facilitar los medios para la operación del servicio a través de la corrupción o evasión legal de requisitos, con una baja exigencia de profesionalización del servicio y poca capacitación a los choferes, no existe un proceso definido. Los mecanismos de control en cuanto a la forma y tiempos en que operan las rutas permiten que en los tramos puedan trabajar de manera autocrática.

Las rutas son trazadas con base en cálculos meramente económicos, cuando la lógica del servicio dice que aquellas deberían establecerse de acuerdo a la demanda de los usuarios para beneficiar a un sector importante de la población. Sin embargo, al no ser así, los camiones urbanos recorren largas distancias para ofrecer el servicio, por lo que el usuario se ve obligado a desplazarse al punto de recolección más cercano a su domicilio. 


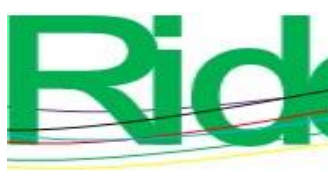

Revista Iberoamericana para la
nvestigación y el Desarrollo Educativo
ISSN $2007-7467$

Por todo lo anterior, surge la necesidad de analizar los indicadores de calidad en el transporte público urbano para que éste pueda ser considerado como una opción de movilidad que satisfaga los nuevos retos de demanda, cobertura y suficiencia establecidos por la dinámica social.

Dicho esto, la identificación de factores determinantes de la satisfacción del servicio de transporte público en el corredor de estudio se realizó en dos momentos: 1) revisión de literatura y 2) diseño de una encuesta para valorar las variables mencionadas.

A partir de la revisión de la literatura, se obtuvo un inventario de factores basado en las aportaciones de Morton et al. (2016) y Backhoff y Vázquez (2002). De esta forma se consideraron 13 criterios: servicio, estancia, personal, espacio, unidades, tiempo, distancia, horario, precio, personal, infraestructura, planeación y manejo.

\section{Metodología}

Una vez revisada la literatura, se determinaron los constructos de la calidad del servicio del TP, a partir de la cual se diseñó una encuesta de 13 ítems como indicadores de calidad de servicio para conocer las experiencias percibidas por los clientes del estado de Colima. La medición de este indicador se realizó a través de una escala tipo Likert de cinco puntos que va desde muy en desacuerdo hasta muy de acuerdo. En dicha encuesta se les pidió a los usuarios de autobuses que indicaran el grado de acuerdo con cada una de las afirmaciones ofrecidas. Los indicadores se detallan en la tabla 1, donde se indican las declaraciones de opinión y una serie de estadísticas descriptivas vinculadas con las respuestas a estas afirmaciones. 


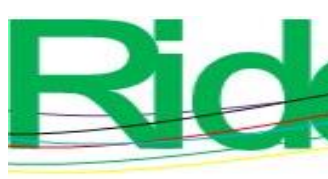

Revista Iberoamericana para la Investigación y el Desarrollo Educativo ISSN $2007-7467$

Tabla 1. Descripción de los constructos y los ítems del instrumento aplicado

\begin{tabular}{|l|l|}
\hline \multicolumn{1}{|c|}{ Constructos } & \multicolumn{1}{|c|}{ Ítem } \\
\hline Variable dependiente: & \\
\hline Satisfacción del servicio & Usaría más el transporte público. \\
\hline Variables independientes & \multicolumn{1}{|c|}{} \\
\hline Horario & $\begin{array}{l}\text { El horario de las rutas es congruente a sus } \\
\text { necesidades. }\end{array}$ \\
\hline Precio & El precio resulta accesible. \\
\hline Confianza del personal & El personal inspira confianza. \\
\hline Servicio & El servicio cubre todas sus necesidades. \\
\hline Estancia & La estancia en la unidad es grata. \\
\hline Atención del personal & El personal atiende siempre sus señales de parada. \\
\hline Espacio & El espacio de las unidades es suficiente y adecuado. \\
\hline Unidades & Las unidades se encuentran en buen estado. \\
\hline Tiempo & El tiempo de espera es el adecuado para usted. \\
\hline Distancia & La distancia de recorrido es la apropiada para usted. \\
\hline Infraestructura & $\begin{array}{l}\text { La infraestructura de las calles está en buenas } \\
\text { condiciones. }\end{array}$ \\
\hline Planeación & $\begin{array}{l}\text { La planeación de recorrido de rutas les permite } \\
\text { acceso a todas las colonias. }\end{array}$ \\
\hline Riesgo & $\begin{array}{l}\text { La rapidez del manejo de la unidad no pone en riesgo } \\
\text { al pasajero. }\end{array}$ \\
\hline
\end{tabular}

Fuente: Elaboración propia

La muestra se determinó en 456 estudiantes de educación media superior y superior, que fueran usuarios del transporte público. A ellos se les aplicó la encuesta diseñada distribuyendo proporcionalmente el número de encuestas en las cuatro ciudades principales del estado de Colima: Colima-Villa de Álvarez (al ser esta una zona conurbada, la información se organizó como una sola ciudad); Tecomán y Manzanillo. La tabla 2 muestra esta distribución de porcentajes de la muestra.

Tabla 2. Características de la muestra

\begin{tabular}{|l|c|c|c|c|c|}
\hline \multicolumn{1}{|c|}{ Ciudad } & $\#$ & $\%$ & Sexo & $\#$ & $\%$ \\
\hline Colima y VA & 197 & 43 & Hombre & 224 & 49 \\
\hline Tecomán & 100 & 22 & Mujer & 232 & 51 \\
\hline Manzanillo & 159 & 35 & & & \\
\hline \multicolumn{1}{|c|}{ Total } & 456 & 100 & Total & & 100 \\
\hline
\end{tabular}

Fuente: Elaboración propia 

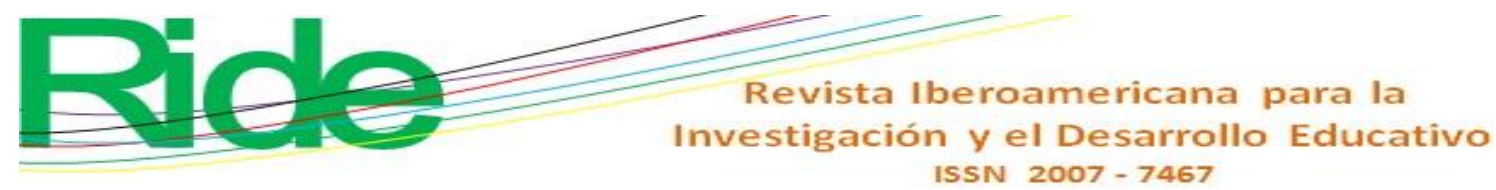

La encuesta fue de elaboración propia y se validó la consistencia interna con la técnica de alfa de Cronbach, en la cual se obtuvo una calificación de 0.81, lo que representa una muy buena consistencia interna (Oviedo y Campo-Arias, 2005; Soler y Soler, 2012).

Para el análisis factorial se aplicaron técnicas estadísticas de ajuste de los modelos de elección discreta para identificar, ponderar y valorar los factores que determinan la elección de la satisfacción de un servicio de transporte colectivo. Al respecto, fue necesario especificar la función de utilidad por estimar con la finalidad de determinar su significatividad, obtener su peso relativo, así como la disponibilidad al pago por una eventual modificación. En este sentido, y considerando que el diseño metodológico incluía solo dos alternativas, se ajustaron modelos de utilidad aleatoria logit binarios, cuya especificación de la función tiene la forma $U_{n}=V_{n}+$ $\varepsilon$.

En términos econométricos, $V_{n}$ representa la parte sistemática, cuantificable y percibida de la utilidad; mientras que $\mathcal{E}$ constituye el error aleatorio que se interpreta como la parte de la utilidad que el modelador no puede medir (Narayan, Cats, van Oort y Hoogendoorn, 2020). El componente $V_{n}$ se especifica con base en las características cuantificables $X_{n}$, que tienen un peso $\beta_{n}^{n}$.

Para esta investigación $V_{n}$ está integrado por horario, precio, confianza del personal, servicio, estancia, atención del personal, espacio, unidades, tiempo, distancia, infraestructura, planeación y riesgo. Su representación matemática se muestra en la ecuación 1:

$V_{i}=\beta_{0}+\beta_{1}$ horario $+\beta_{2}$ precio $+\beta_{3}$ confianza del pesonal $+\beta_{4}$ servicio + $\beta_{5}$ estancia $+\beta_{6}$ atención del personal $+\beta_{7}$ espacio $+\beta_{8}$ unidades $+\beta_{9}$ tiempo + $\beta_{10}$ distancia $+\beta_{11}$ infraestructura $+\beta_{12}$ planeación $+\beta_{13}$ riesgo (ecuación 1)

La ecuación anterior representa la utilidad (satisfacción) percibida por el usuario para elegir en cuáles condiciones utilizar más el transporte colectivo. Los factores ligados a nivel de servicio se trataron como variables continuas, mientras que el sexo, la ciudad y la ocupación se consideraron como variables categóricas.

Los parámetros $\beta_{i}$ surgen del ajuste econométrico de la razón máxima verosimilitud. Se parte de la existencia de una constante específica $\beta_{0}$, que es el aumento del uso del transporte colectivo como una alternativa mejorada. Esto debido a que las encuestas sitúan al usuario en una elección hipotética en la que aumentaría el uso del servicio de TP.

Esta constante permite capturar, por un lado, el conjunto de mejoras del servicio no observables por el encuestado, pero que lo llevan a imaginar una alternativa de servicio; por otro 


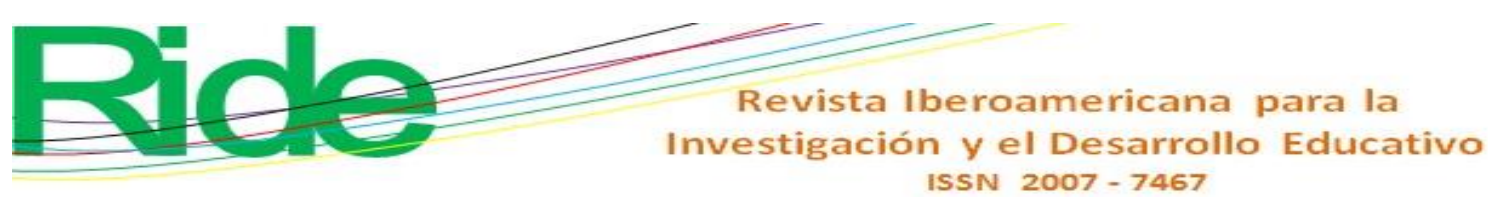

lado, las brechas de satisfacción entre las expectativas del servicio y sus condiciones reales, lo que permite a los responsables del servicio público tomar cartas en el asunto para su mejora.

\section{Resultados}

Siguiendo la técnica de análisis exploratorio, la tabla 3 muestra la correlación de los 13 indicadores de la calidad del servicio identificados. 


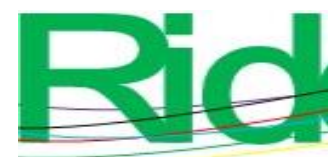

Tabla 3. Correlación de los factores del servicio de transporte público

\begin{tabular}{|c|c|c|c|c|c|c|c|c|c|c|c|c|c|c|}
\hline & $\begin{array}{l}\text { Uti } \\
\text { lid } \\
\text { ad }\end{array}$ & $\begin{array}{l}\text { Ho } \\
\text { ra }\end{array}$ & $\begin{array}{l}\text { Pre } \\
\text { cio }\end{array}$ & $\begin{array}{l}\text { Per } \\
\text { son } \\
\text { al }\end{array}$ & $\begin{array}{l}\text { Ser } \\
\text { vic } \\
\text { io }\end{array}$ & $\begin{array}{l}\text { Est } \\
\text { anc } \\
\text { ia }\end{array}$ & $\begin{array}{l}\text { Per } \\
\text { son } \\
\text { al }\end{array}$ & $\begin{array}{l}\text { Es } \\
\text { pac } \\
\text { io }\end{array}$ & $\begin{array}{l}\text { Un } \\
\text { ida } \\
\text { des }\end{array}$ & $\begin{array}{l}\text { Tie } \\
\text { mp } \\
\text { o }\end{array}$ & $\begin{array}{l}\text { Dist } \\
\text { anci } \\
\text { a }\end{array}$ & $\begin{array}{l}\text { Inf } \\
\text { rae } \\
\text { s } \\
\text { tru } \\
\text { ctu } \\
\text { ra }\end{array}$ & $\begin{array}{l}\text { Pla } \\
\text { nea } \\
\text { ció } \\
\text { n }\end{array}$ & $\begin{array}{l}\mathrm{Ri} \\
\text { es } \\
\text { go }\end{array}$ \\
\hline Utilidad & $\begin{array}{l}1.0 \\
00\end{array}$ & & & & & & & & & & & & & \\
\hline $\begin{array}{l}\text { Horario } \\
\text { congruen } \\
\text { te }\end{array}$ & $\begin{array}{l}.45 \\
39 \\
* \\
\end{array}$ & $\begin{array}{l}1.0 \\
00\end{array}$ & & & & & & & & & & & & \\
\hline $\begin{array}{l}\text { Precio } \\
\text { accesible }\end{array}$ & $\begin{array}{l}.53 \\
50 \\
*\end{array}$ & $\begin{array}{l}.49 \\
35 \\
*\end{array}$ & $\begin{array}{l}1.0 \\
00\end{array}$ & & & & & & & & & & & \\
\hline $\begin{array}{l}\text { Personal } \\
\text { inspira } \\
\text { confianz } \\
\text { a }\end{array}$ & $\begin{array}{l}.40 \\
24 \\
*\end{array}$ & $\begin{array}{l}.44 \\
58 \\
*\end{array}$ & $\begin{array}{l}.50 \\
42 \\
*\end{array}$ & $\begin{array}{l}1.0 \\
00\end{array}$ & & & & & & & & & & \\
\hline $\begin{array}{l}\text { Servicio } \\
\text { cubre } \\
\text { necesida } \\
\text { d }\end{array}$ & $\begin{array}{l}.43 \\
95 \\
*\end{array}$ & $\begin{array}{l}.49 \\
74 \\
*\end{array}$ & $\begin{array}{l}.48 \\
31 \\
*\end{array}$ & $\begin{array}{l}.64 \\
19 \\
*\end{array}$ & $\begin{array}{l}1.0 \\
00\end{array}$ & & & & & & & & & \\
\hline $\begin{array}{l}\text { Estancia } \\
\text { agradabl } \\
\text { e }\end{array}$ & $\begin{array}{l}44 \\
31 \\
*\end{array}$ & $\begin{array}{l}.41 \\
93 \\
*\end{array}$ & $\begin{array}{l}.43 \\
95 \\
*\end{array}$ & $\begin{array}{l}62 \\
04 \\
*\end{array}$ & $\begin{array}{l}.65 \\
46 \\
*\end{array}$ & $\begin{array}{l}1.0 \\
00\end{array}$ & & & & & & & & \\
\hline $\begin{array}{l}\text { Personal } \\
\text { atiende }\end{array}$ & $\begin{array}{l}.40 \\
33 \\
*\end{array}$ & $\begin{array}{l}.41 \\
34 \\
*\end{array}$ & $\begin{array}{l}.40 \\
48 \\
*\end{array}$ & $\begin{array}{l}.49 \\
87 \\
*\end{array}$ & $\begin{array}{l}.51 \\
96 \\
*\end{array}$ & $\begin{array}{l}.54 \\
44 \\
*\end{array}$ & $\begin{array}{l}1.0 \\
00\end{array}$ & & & & & & & \\
\hline $\begin{array}{l}\text { Espacio } \\
\text { suficient } \\
\mathrm{e}\end{array}$ & $\begin{array}{l}.37 \\
28 \\
*\end{array}$ & $\begin{array}{l}.43 \\
30 \\
* \\
\end{array}$ & $\begin{array}{l}.41 \\
04 \\
*\end{array}$ & $\begin{array}{l}.48 \\
42 \\
* \\
\end{array}$ & $\begin{array}{l}.52 \\
29 \\
* \\
\end{array}$ & $\begin{array}{l}.59 \\
26 \\
*\end{array}$ & $\begin{array}{l}.57 \\
06 \\
*\end{array}$ & $\begin{array}{l}1.0 \\
00\end{array}$ & & & & & & \\
\hline $\begin{array}{l}\text { Unidades } \\
\text { en buen } \\
\text { estado }\end{array}$ & $\begin{array}{l}\cdot \\
58 \\
19 \\
*\end{array}$ & $\begin{array}{l}.38 \\
30 \\
*\end{array}$ & $\begin{array}{l}.37 \\
28 \\
*\end{array}$ & $\begin{array}{l}.49 \\
76 \\
*\end{array}$ & $\begin{array}{l}.46 \\
03 \\
*\end{array}$ & $\begin{array}{l}.52 \\
61 \\
*\end{array}$ & $\begin{array}{l}.46 \\
85 \\
*\end{array}$ & $\begin{array}{l}.58 \\
19 \\
*\end{array}$ & $\begin{array}{l}1.0 \\
00\end{array}$ & & & & & \\
\hline $\begin{array}{l}\text { Tiempo } \\
\text { de espera }\end{array}$ & $\begin{array}{l}.47 \\
42\end{array}$ & $\begin{array}{l}.51 \\
29 \\
*\end{array}$ & $\begin{array}{l}.36 \\
73 \\
*\end{array}$ & $\begin{array}{l}.48 \\
54 \\
*\end{array}$ & $\begin{array}{l}.47 \\
27 \\
*\end{array}$ & $\begin{array}{l}.41 \\
06 \\
*\end{array}$ & $\begin{array}{l}.42 \\
86 \\
*\end{array}$ & $\begin{array}{l}.50 \\
59 \\
*\end{array}$ & $\begin{array}{l}.56 \\
49 \\
*\end{array}$ & $\begin{array}{l}1.0 \\
00\end{array}$ & & & & \\
\hline $\begin{array}{l}\text { Distancia } \\
\text { del } \\
\text { recorrido }\end{array}$ & $\begin{array}{l}.52 \\
29 \\
* \\
\end{array}$ & $\begin{array}{l}.39 \\
82 \\
* \\
\end{array}$ & $\begin{array}{l}.48 \\
36 \\
* \\
\end{array}$ & $\begin{array}{l}.49 \\
48 \\
* \\
\end{array}$ & $\begin{array}{l}.52 \\
28 \\
* \\
\end{array}$ & $\begin{array}{l}.46 \\
88 \\
* \\
\end{array}$ & $\begin{array}{l}.47 \\
46 \\
* \\
\end{array}$ & $\begin{array}{l}.43 \\
03 \\
* \\
\end{array}$ & $\begin{array}{l}.52 \\
84 \\
* \\
\end{array}$ & $\begin{array}{l}.63 \\
13 \\
* \\
\end{array}$ & $\begin{array}{l}1.00 \\
0\end{array}$ & & & \\
\hline $\begin{array}{l}\text { Infraestr } \\
\text { uctura } \\
\text { carretera }\end{array}$ & $\begin{array}{l}.53 \\
74 \\
* \\
\end{array}$ & $\begin{array}{l}.36 \\
08 \\
* \\
\end{array}$ & $\begin{array}{l}.43 \\
42 \\
* \\
\end{array}$ & $\begin{array}{l}.49 \\
38 \\
* \\
\end{array}$ & $\begin{array}{l}.42 \\
79 \\
* \\
\end{array}$ & $\begin{array}{l}.48 \\
13 \\
* \\
\end{array}$ & $\begin{array}{l}.46 \\
63 \\
* \\
\end{array}$ & $\begin{array}{l}.50 \\
53 \\
*\end{array}$ & $\begin{array}{l}.51 \\
14 \\
*\end{array}$ & $\begin{array}{l}.54 \\
06 \\
*\end{array}$ & $\begin{array}{l}.567 \\
7 *\end{array}$ & $\begin{array}{l}1.0 \\
00\end{array}$ & & \\
\hline $\begin{array}{l}\text { Planeaci } \\
\text { ón del } \\
\text { recorrido }\end{array}$ & $\begin{array}{l}.55 \\
50 \\
*\end{array}$ & $\begin{array}{l}.44 \\
72 \\
*\end{array}$ & $\begin{array}{l}.49 \\
30 \\
*\end{array}$ & $\begin{array}{l}.54 \\
40 \\
*\end{array}$ & $\begin{array}{l}.46 \\
49 \\
*\end{array}$ & $\begin{array}{l}.43 \\
20 \\
*\end{array}$ & $\begin{array}{l}.43 \\
01 \\
*\end{array}$ & $\begin{array}{l}.43 \\
47 \\
*\end{array}$ & $\begin{array}{l}.47 \\
42 \\
*\end{array}$ & $\begin{array}{l}.52 \\
29 \\
*\end{array}$ & $\begin{array}{l}.537 \\
4^{*}\end{array}$ & $\begin{array}{l}.55 \\
50 \\
*\end{array}$ & $\begin{array}{l}1.0 \\
00\end{array}$ & \\
\hline
\end{tabular}




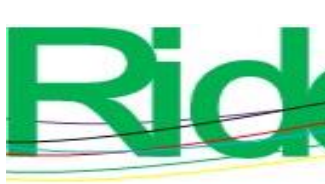

Revista Iberoamericana para la Investigación y el Desarrollo Educativo ISSN $2007-7467$

De forma general, en este modelo se obtuvieron coeficientes con niveles de significancia correctos. Los factores considerados como determinantes de la calidad del servicio resultaron con tendencias positivas; esto sugiere que al incrementarse impacta el nivel de la satisfacción de los usuarios.

Por otro lado, los factores relacionados con el tiempo y la distancia del recorrido resultaron negativos, lo que indica que un incremento de estos factores se transforma en una insatisfacción, al decrecer la calidad percibida por usuario. Los factores considerados son significativos a intervalos de confianza de $99 \%$ y $95 \%$.

Del modelo anterior, se puede observar que el factor precio accesible obtiene el coeficiente más alto y además positivo, lo que incrementa 2.09 veces más la probabilidad de satisfacción del usuario. Seguido de este se ubica el horario de las rutas, el cual incrementa 1.7 veces más dicha probabilidad. Posteriormente, surge el factor subjetivo el personal inspira confianza (1.40 veces más), la planeación del recorrido (1.33 veces más), el servicio cubre las necesidades (1.25 veces más) y la infraestructura carretera (1.23 veces más).

En términos absolutos, el precio del viaje, seguido de la planeación, tienen mayor peso en su efecto sobre el valor de la satisfacción por dos razones: costo y recorrido. El primer factor tiene relación con el costo pagado y el valor percibido del servicio, en el cual su efecto en la satisfacción percibida es el más importante. El segundo se asocia con la planeación del recorrido, e implica a su vez otros factores como el tiempo de espera y la distancia del recorrido, los cuales resultaron con un efecto negativo sobre la satisfacción.

Con base en los resultados, el incremento en la satisfacción del servicio (utilidad) sin el aumento del precio del transporte se relaciona con la implementación de medidas que ofrezcan un aspecto más formal, lo que generaría más confianza en los usuarios. De igual manera, la planeación de los recorridos aumentaría la congruencia de los horarios, así como la cobertura de las necesidades, lo que reduciría el tiempo de espera y la distancia del recorrido.

Desde la perspectiva económica, este aumento de la satisfacción del usuario exige mayor inversión, así como ajustes de orden estratégico y táctico en la planeación del sistema de transporte público en Colima. 


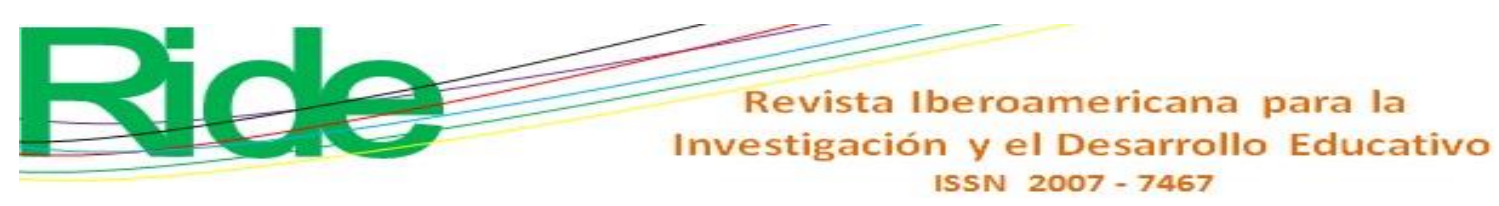

\section{Conclusiones}

El objetivo de esta investigación fue estimar los factores que intervienen en la percepción de la calidad de servicio del transporte público urbano en el estado de Colima. La escala que se diseñó para tal efecto comprendió 13 factores: horario congruente, precio accesible, personal inspira confianza, servicio cubre necesidad, estancia agradable, personal atiende, espacio suficiente, unidades en buen estado, tiempo de espera, distancia del recorrido, infraestructura carretera, planeación del recorrido y riesgo del manejo.

Los resultados enseñan que, desde la satisfacción para los usuarios, los factores que resultaron significativos fueron los siguientes: horario congruente, precio accesible, personal inspira confianza, servicio cubre las necesidades, tiempo de espera, distancia del recorrido, infraestructura carretera y planeación del recorrido, los cuales presentan mayor probabilidad de incidencia en la calidad del servicio del transporte público.

Igualmente, de forma paralela se estimaron los factores que intervienen en la percepción de la calidad de servicio del transporte colectivo urbano en el estado de Colima, así como la aplicación de un modelo de utilidad de elección discreta para ponderar y valorar los factores que impacta en la calidad del servicio. En tal sentido, la importancia dada por las diferentes ciudades analizadas, así como el efecto del sexo sobre los factores estadísticamente significativos proveen información de decisión para que los responsables estatales y municipales establezcan medidas generales para gestionar mejores estrategias del TP en cada ciudad analizada. De hecho, los resultados del análisis efectuado mostraron las implicaciones de estos datos para un estudio posterior sobre las necesidades y las mejoras que se pueden emprender en cuanto a movilidad.

Esto último no implicaría un aumento de la tarifa o de las utilidades económicas, sino un incremento en la satisfacción de los usuarios del TP. Sin embargo, es importante tomar en cuenta el efecto de representatividad de la población, pues los datos analizados corresponden a las particularidades de los estudiantes del estado de Colima.

De forma general, esta investigación reflejó el valor agregado que se puede obtener a través de un análisis más exhaustivo de los datos existentes sobre los servicios ofertados a los estudiantes universitarios. De hecho, con el aumento de los datos disponibles surgen importantes oportunidades para trabajos posteriores que podrían partir de datos secundarios que tengan una relación directa con los agentes sociales relacionados con la elaboración de políticas públicas. 


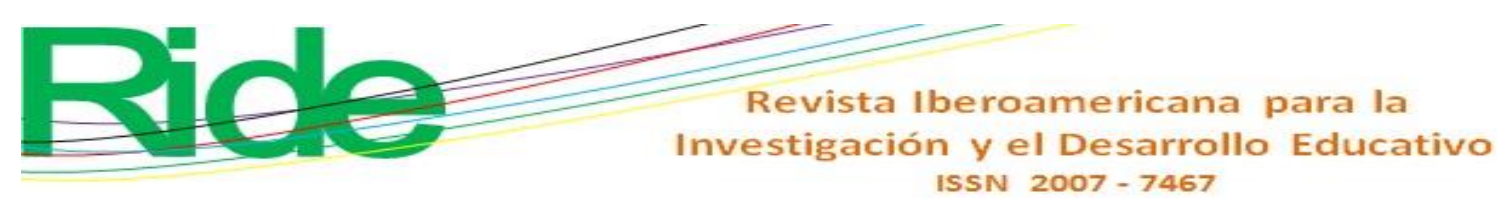

Futuras líneas de investigación

La principal limitante de esta investigación se encuentra en que sus resultados (al ser un estudio no probabilístico) no muestran de manera representativa a todos los usuarios del transporte público en el estado de Colima. Además, esta fue una indagación desarrollada con énfasis en el punto de vista estudiantil, y no de los directivos.

Por eso, se pueden abarcar otros perfiles de usuarios para corroborar o no los resultados mostrados. Asimismo, una futura línea de investigación puede tomar en cuenta que los resultados de la presente investigación muestran la presencia de una subvaloración sobre la percepción del precio a pagar por los usuarios en relación con las ciudades y el sexo. Esto sugiere que las estrategias de mejora deben implementarse con reservas por sus implicaciones en el rubro de la inversión, así como de ajustes de planeación del sistema de transporte público en Colima. En otras palabras, este modelo puede establecer un diseño metodológico propio en función del contexto nacional.

\section{Referencias}

Aparicio, S., Ramírez, A. y Gómez, D. (2013). Elección de ocupaciones que generen empleo usando modelos de elección discreta: Medellín Área Metropolitana 2009. Estudios Gerenciales, 29(129), 476-484. Doi: https://doi.org/10.1016/j.estger.2013.11.013

Backhoff, M. Á. y Vázquez, J. C. (2002). El sistema de información geoestadística para el transporte: métodos, organización y descripción operativa. Instituto Mexicano del Transporte.

Recuperado http://www.imt.mx/archivos/Publicaciones/PublicacionTecnica/pt207.pdf

Becerra, F., Serna, H. y Naranjo, J. (2013). Redes empresariales locales, investigación y desarrollo e innovación en la empresa. Cluster de herramientas de Caldas, Colombia. $\begin{array}{llll}\text { Estudios } & \text { Gerenciales, } & \text { 29(127), }\end{array}$ https://doi.org/10.1016/j.estger.2013.05.013

Callejas-Cuervo, M., Valero-Bustos, H., y Alarcón-Aldana, A. (2014). Agentes de software como herramienta para medir la calidad de servicio prestado en un sistema de transporte público colectivo urbano. Información Tecnológica, 25(5), 147-154. Doi: https://doi.org/10.4067/S0718-07642014000500020

Cascetta, E. y Cartenì, A. (2011). A Quality-Based Approach to Public Transportation Planning: Theory and a Case Study. International Journal of Sustainable Transportation, 8(1), 84106. Doi: https://doi.org/10.1080/15568318.2012.758532 


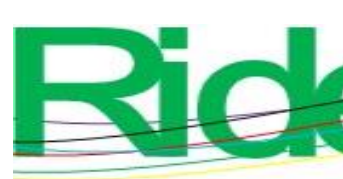

Revista Iberoamericana para la
Investigación y el Desarrollo Educativo
ISSN $2007-7467$

Duque, E. y Gómez, Y. (2015). Evolución conceptual de los modelos de medición de la percepción de calidad del servicio: una mirada desde la educación superior. Suma de Negocios, 5(12), 180-191. Doi: https://doi.org/doi:10.1016/S2215-910X(14)70040-0

Guercio, B., Martínez, L. y Vigier, H. (2017). Las limitaciones al financiamiento bancario de las pymes de alta tecnología. Estudios Gerenciales, 33(142), 3-12. Doi: https://doi.org/doi:10.1016/j.estger.2017.02.001

Morton, C., Caulfield, B. and Anable, J. (2016). Customer perceptions of quality of service in public transport: Evidence for bus transit in Scotland. Case Studies on Transport Policy, 4(3), 199-207. Doi: https://doi.org/10.1016/j.cstp.2016.03.002

Narayan, J., Cats, O., van Oort, N. and Hoogendoorn, S. (2020). Integrated route choice and assignment model for fixed and flexible public transport systems. Transportation Research Part C: Emerging Technologies, 115(10), 26-31. Doi: https://doi.org/doi:10.1016/j.trc.2020.102631

Oviedo, H. y Campo-Arias, A. (2005). Aproximación al uso del coeficiente alfa de Cronbach. Revista Colombiana de Psiquiatría, 34(4), 572-580.

Periódico Oficial del Estado de Colima [POEC] (2017). Ley de Movilidad Sustentable para el Estado de Colima. Recuperado de https://congresocol.gob.mx/web/Sistema/uploads/LegislacionEstatal/LeyesEstatales/M ovilidad_Sustentable30ene2017.pdf

Rodríguez, J. y Díaz, J. (2014). Evaluación de impacto del sistema de transporte Metrolínea: revisión de metodologías. Equidad \& Desarrollo, 22(1), 121-135. Recuperado de http://revistas.lasalle.edu.co/index.php/ed/article/view/3253

Román, C., Martín, J. and Espino, R. (2014). Using Stated Preferences to Analyze the Service Quality of Public Transport. International Journal of Sustainable Transportation, 8(1), 28-46. Doi: https://doi.org/10.1080/15568318.2012.758460

Sánchez-Flores, O. y Romero-Torres, J. (2010). Factores de calidad del servicio en el transporte público de pasajeros: estudio de caso de la ciudad de Toluca, México. Economía, Sociedad y Territorio, 10(32), 49-80. Doi: https://doi.org/10.22136/est002010152

Soler, S. y Soler, L. (2012). Usos del coeficiente alfa de Cronbach en el análisis de instrumentos escritos. Revista Médica Electrónica, 34(1), 01-06.

Sottile, E., Di Teulada, B., Meloni, I. and Cherchi, E. (2018). Estimation and validation of hybrid choice models to identify the role of perception in the choice to cycle. International 


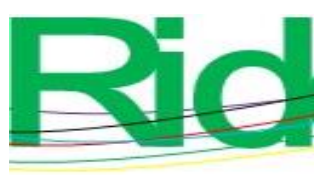

Revista Iberoamericana para la Investigación y el Desarrollo Educativo ISSN $2007-7467$

Journal of Sustainable Transportation, 13(8), 543-552. Doi: https://doi.org/10.1080/15568318.2018.1490465

Stathopoulos, A. and Marcucci, E. (2014). De Gustibus Disputandum Est: Non-Linearity in Public Transportation Service Quality Evaluation. International Journal of Sustainable Transportation, 8(1), 47-68. Doi: https://doi.org/0.1080/15568318.2012.758523

Subramaniana, N., Gunasekaran, A., Yu, J., Cheng, J. and Ning, K. (2014). Customer satisfaction and competitiveness in the Chinese E-retailing: Structural equation modeling (SEM) approach to identify the role of quality factors. Expert Systems with Applications, 41(1), 69-80. Doi: https://doi.org/10.1016/j.eswa.2013.07.012

Torres, J. (2011). Posibilidades, logros y desafíos en la implementación de modelos de calidad en los gobiernos latinoamericanos. Estudios Gerenciales, 27(11), 33-57. Doi: https://doi.org/10.1016/S0123-5923(11)70156-5

Varian, H. (1982). The Nonparametric Approach to Demand Analysis. Econometria, 50(4), 945973. Doi: https://doi.org/10.2307/1912771 


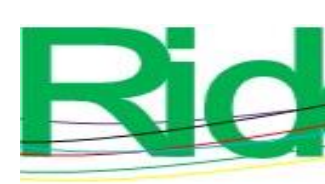

Revista Iberoamericana para la Investigación y el Desarrollo Educativo

ISSN 2007 - 7467

\begin{tabular}{|l|ll|}
\hline Rol de Contribución & Definición (solo poner nombre del autor) \\
\hline Conceptualización & $\begin{array}{l}\text { Omar Alejandro Pérez Cruz(principal)-Rogelio Pinto } \\
\text { Pérez(igual) }\end{array}$ \\
\hline Metodología & Omar Alejandro Pérez Cruz & \\
\hline Software & Omar Alejandro Pérez Cruz & \\
\hline Validación & Rogelio Pinto Pérez & \\
\hline Análisis Formal & Omar Alejandro Pérez Cruz & \\
\hline Investigación & Rogelio Pinto Pérez & \\
\hline Recursos & Rogelio Pinto Pérez & \\
\hline Curación de datos & Omar Alejandro Pérez Cruz & \\
\hline $\begin{array}{l}\text { Escritura - Preparación del } \\
\text { borrador original }\end{array}$ & Omar Alejandro Pérez Cruz & \\
\hline Escritura - Revisión y edición & $\begin{array}{l}\text { Omar Alejandro Pérez Cruz(principal)-Rogelio } \\
\text { Pérez(apoya) }\end{array}$ \\
\hline Visualización & $\begin{array}{l}\text { Omar Alejandro Pérez Cruz(principal)-Rogelio Pinto } \\
\text { Pérez(apoya) }\end{array}$ & \\
\hline Supervisión & $\begin{array}{l}\text { Omar Alejandro Pérez Cruz(principal)-Rogelio Pinto } \\
\text { Pérez(apoya) }\end{array}$ & \\
\hline Administración de Proyectos & Rogelio Pinto Pérez & \\
\hline Adquisición de fondos & Rogelio Pinto Pérez & \\
\hline
\end{tabular}

